\title{
Panorama brasileiro dos serviços de plantas medicinais e fitoterápicos
}

Brazilian panorama of medicinal and phytotherapeutic plants services

Panorama brasileño de los servicios de plantas medicinales y de los medicamentos a base de hierbas

\section{Marcio Rossato Badke', Silvana Bastos Cogo"I , Aline Gomes Ilha' ${ }^{\mathrm{III}}$, Elisa Vanessa Heisler ${ }^{\mathrm{IV}}$, Maria Denise Schimith ${ }^{\mathrm{v}}$, Henriqueta Tereza do Sacramento ${ }^{\mathrm{VI}}$}

\begin{abstract}
Resumo: Objetivo: verificar os estabelecimentos de saúde credenciados no Sistema Único de Saúde e no Cadastro Nacional de Estabelecimentos de Saúde, que utilizam plantas medicinais e fitoterápicos como Práticas Integrativas e Complementares. Método: pesquisa documental no Cadastro Nacional de Estabelecimentos de Saúde e em leis, portarias e resoluções brasileiras referentes às Práticas Integrativas e Complementares em maio de 2018, por meio da análise de conteúdo. Resultados: dos 5.570 municípios brasileiros, 126 apresentam algum serviço que utilizavam a fitoterapia como prática integrativa e complementar. Em 110 municípios, os serviços estão na rede de atenção do Sistema Único de Saúde, distribuídos em 563 serviços que utilizam a fitoterapia. Conclusões: o estudo permitiu conhecer a realidade brasileira sobre a prestação legal do serviço de fitoterapia, subsidiando os gestores no planejamento, monitoramento e avaliação dos cadastros e do funcionamento destes serviços.
\end{abstract}

Descritores: Fitoterapia; Plantas medicinais; Enfermagem; Conhecimento; Política pública

Abstract: Aim: to verify the health establishments accredited in the Unified Health System and in the National Register of Health Establishments, which use herbal and phytotherapeutic plants as Integrative and Complementary Practices. Method: documentary research in the National Register of Health Establishments and in Brazilian laws, ordinances and resolutions referring to Integrative and Complementary Practices in May 2018, through content analysis. Results: of the 5,570 Brazilian municipalities, 126 present some service that use

\footnotetext{
${ }^{\mathrm{I}}$ Enfermeiro. Doutor em Ciências pela UFPel. Professor Adjunto do Departamento de Enfermagem da Universidade Federal de Santa Maria, RS, Brasil. Bolsista de Doutorado Sanduíche no Exterior do CNPq (2015/2016). E-mail: marciobadke@gmail.com ou lapics@ufsm.br ORCID: https://orcid.org/0000-0002-9459-1715

II Enfermeira. Doutora em Enfermagem pela FURG. Professora Adjunta do Departamento de Enfermagem da Universidade Federal de Santa Maria, RS, Brasil. E-mail: silvanabastoscogo@gmail.com ORCID: https://orcid.org/0000-0002-1686-8459

III Discente do curso de Enfermagem da Universidade Federal de Santa Maria, RS, Brasil. Bolsista FIPE JR- CCS 2019. E-mail: aline.gomes1996@hotmail.com ORCID: https://orcid.org/0000-0002-5715-9595

IV Enfermeira. Mestre em enfermagem pela Universidade Federal de Santa Maria, RS, Brasil. E-mail: elisa.vanessa@yahoo.com.br ORCID: https://orcid.org/0000-0001-5438-0983

${ }^{v}$ Enfermeira. Doutora em Ciências pela Unifesp. Professora Adjunta do Departamento de Enfermagem da Universidade Federal de Santa Maria, RS, Brasil. E-mail: ma.denise2011@gmail.com ORCID: https://orcid.org/0000-0002-4867-4990

${ }^{V I}$ Médica. Mestre em Políticas Públicas e Desenvolvimento local pela Escola Superior de Ciências da Santa Casa de Misericórdia de Vitória, ES, Brasil. E-mail: htsacramento1982@gmail.com ORCID: https://orcid.org/0000-0002-7564-560X
} 
phytotherapy as an integrative and complementary practice. In 110 municipalities the services are in the care network of the Unified Health System, distributed into 563 services that use phytotherapy. Conclusions: the study allowed the knowledge on the Brazilian reality about the legal provision of the phytotherapy service, subsidizing the managers in the planning, monitoring and evaluation of the registries and the operation of these services.

Descriptors: Phytotherapy; Plants, medicinal; Nursing; Knowledge; Public policy

Resumen: Objetivo: verificar los establecimientos de salud acreditados en el Sistema Único de Salud y en el Catastro Nacional de Establecimientos de Salud, que utilizan plantas medicinales y de los medicamentos a base de hierbas como Prácticas Integrativas y Complementarias. Método: investigación documental en el Catastro Nacional de Establecimientos de Salud y en leyes, decretos y resoluciones brasileñas referentes a las Prácticas Integrativas y Complementarias en mayo de 2018, con análisis de contenido. Resultados: de los 5.570 municipios brasileños, 126 presentan algún servicio que utilizan los medicamentos a base de hierbas como práctica integrativa y complementaria. En 110 municipios los servicios están en la red de atención del Sistema Único de Salud, distribuidos en 563 servicios que utilizan la fitoterapia. Conclusiones: el estudio permitió conocer la realidad brasileña sobre la prestación legal del uso de medicamentos a base de hierbas, subsidiando a los gestores en la planificación, el monitoreo y la evaluación de los registros y del funcionamiento de estos servicios.

Descriptores: Medicamentos a base de hierbas; Plantas medicinales; Enfermería; Conocimiento; Política pública

\section{Introdução}

O consumo popular de plantas medicinais e seus derivados como prática terapêutica para saúde tem seus primeiros registros desde o início da civilização, sendo técnica de cuidado integrante do campo das medicinas populares e dos saberes ancestrais. ${ }^{1}$ A medicina alopática vem se destacando no mercado farmacêutico, entretanto, a aplicação de plantas medicinais e fitoterápicos ainda é amplamente utilizada pela população devido, principalmente, aos efeitos colaterais causados por medicamentos sintéticos, demonstrando melhor adesão ao consumo de produtos naturais, assim como as Práticas Integrativas e Complementares (PICS), com abordagens holísticas aplicadas aos conceitos de saúde e bem-estar. ${ }^{2}$

A fitoterapia é a terapêutica caracterizada pelo uso de plantas medicinais em suas diferentes formas farmacêuticas, sem a utilização de substâncias ativas isoladas, ainda que de origem vegetal. ${ }^{3}$ Desta maneira, a fitoterapia engloba as plantas medicinais e os fitoterápicos.

As plantas medicinais são espécies vegetais, cultivadas ou não, utilizadas com propósitos terapêuticos. As plantas podem ser frescas quando coletadas no momento de seu uso e secas as que foram precedidas de secagem e estabilização, ambas equivalendo à droga vegetal. ${ }^{3}$ Os 
3 | Badke MR, Cogo SB, Ilha AG, Heisler EV, Schimith MD, Sacramento HT

fitoterápicos são medicamentos obtidos exclusivamente de matérias-primas ativas vegetais, cuja eficácia e segurança são validadas. Não é considerado fitoterápico aquele que em sua composição, inclua substâncias ativas isoladas e associações destas com extratos vegetais. ${ }^{4}$

No Brasil, a legitimação e a institucionalização das PICS de atenção à saúde tiveram início na década de 1980, após a criação do Sistema Único de Saúde (SUS). ${ }^{4}$ Em 2002, a Organização Mundial de Saúde (OMS) formulou uma orientação de estímulo à inclusão de práticas não convencionais de cuidado na organização dos sistemas de saúde oficiais, diante da evidência do aumento significativo de uso por parte da população. ${ }^{5}$

Esta orientação da OMS veio somar ao que já era uma reivindicação no Brasil. Na VIII Conferência Nacional de Saúde, em 1986, foi deliberada a introdução de práticas alternativas de assistência no âmbito dos serviços de saúde, possibilitando ao usuário o acesso democrático de escolher a terapêutica preferida. ${ }^{6}$ Ao mesmo tempo, profissionais de saúde e gestores comprometidos com a Reforma Sanitária Brasileira defendiam que as plantas medicinais e fitoterápicas poderiam ser terapêuticas acessíveis, resgatando o saber popular e fortalecendo as terapias naturais, com menos riscos de efeitos colaterais. ${ }^{7}$

Encontros nacionais foram acontecendo para trocas de experiências sobre Fitoterapia no SUS e em 1999, durante o Simpósio Nacional de Plantas medicinais, em Águas de Lindóia, São Paulo, foi fundada a Associação Nacional de Fitoterapia em Serviços Públicos, que trabalhou para o fortalecimento dos programas de fitoterapia e em defesa da institucionalização da Política Nacional de Plantas Medicinais e Fitoterápicas. ${ }^{8}$

A Gerência de Assistência Farmacêutica da Secretaria de Ciência Tecnologia e Insumos, do Ministério da Saúde (MS), iniciou o processo de institucionalização desde 2000, momento que constituiu um Grupo de Trabalho composto de profissionais do SUS, que coordenavam programas de Fitoterapia e pesquisadores para a elaboração da Política Nacional de Plantas 
Medicinais e Medicamentos Fitoterápicos. ${ }^{7}$ Em 2001 e 2003 foram realizados seminários nacionais visando à validação da proposta da Política. ${ }^{7}$

O encaminhamento desta reivindicação passou a ser pauta do MS, especialmente em 2003, quando nomeou uma equipe de trabalho que, em 2004, realizou um diagnóstico junto aos municípios, observando as diferentes práticas terapêuticas oferecidas. Este estudo foi dirigido a 5.560 municípios, obtendo retorno de 4.052 gestores municipais, sendo que destes, $72 \%$ (2.917 municípios) utilizavam PICS na Atenção Básica e 30\% tinham lei ou ato institucional certificando o uso das PICS, predominando municípios da região Sul e Sudeste. ${ }^{9}$

Após a conclusão dos trabalhos foi instituída a Política Nacional de Prática Integrativa e Complementar (PNPIC) no SUS, pela Portaria GM/MS n. 971, de 3 de maio de 2006, que contemplava principalmente as áreas de Fitoterapia e Plantas Medicinais, Termalismo, Antroposofia, Homeopatia e Medicina Tradicional Chinesa (MTC) Acupuntura. ${ }^{10}$ No dia de 22 de junho de 2006, por meio do Decreto Presidencial n. 5.813, foi aprovada a Política Nacional de Plantas Medicinais e Fitoterápicos. ${ }^{9}$

A política das PICS foi apresentada com os objetivos de incorporar, implementar, estruturar e fortalecer as referidas práticas no SUS; contribuir para o aumento da resolubilidade do sistema e para a ampliação do acesso às PICS, particularmente dos medicamentos homeopáticos e fitoterápicos; promover a racionalização das ações de saúde; estimular as ações referentes ao controle/participação social; desenvolver estratégias de qualificação de pessoal; divulgar conhecimentos e informações sobre PICS para profissionais de saúde, gestores e usuários do SUS e incentivar as ações intersetoriais, a pesquisa, as ações de acompanhamento e avaliação, além de cooperação nacional e internacional no âmbito das práticas integrativas e complementares. $^{9}$ 
5 | Badke MR, Cogo SB, Ilha AG, Heisler EV, Schimith MD, Sacramento HT

Em 2017, a PNPIC foi ampliada por meio da inclusão de 14 novas práticas, a partir da publicação da portaria GM 849/2017. ${ }^{11}$ No ano seguinte, em 2018, a PNPIC foi novamente ampliada, a partir da publicação da portaria GM 702/2018, passando a incluir 10 novas práticas. ${ }^{12}$

Para que a política das PICS se concretize, é imprescindível que ocorra o cadastro dos estabelecimentos de saúde, pois propicia ao gestor conhecimento da rede assistencial existente e suas potencialidades, visando auxiliar no planejamento em saúde em todos os níveis de governo. Neste sentido, o MS instituiu o Cadastro Nacional de Estabelecimentos de Saúde (CNES) com o intuito de disponibilizar informações das condições de infraestrutura e funcionamento dos serviços. ${ }^{13}$

O CNES visa ser a base para operacionalizar os Sistemas de Informações em Saúde, sendo estes imprescindíveis para um gerenciamento eficaz e eficiente do SUS. O sistema de informação auxilia o planejamento em saúde, em todos os níveis do governo; disponibiliza informações de infraestrutura, tipo de atendimento prestado, serviços especializados, leitos e profissionais de saúde existentes nos estabelecimentos de saúde. Além disso, propicia ao gestor o conhecimento da realidade da rede assistencial existente, sua potencialidade e capacidade instalada, bem como dar maior visibilidade ao controle social a ser exercido pela população. ${ }^{13}$

Este artigo tem como questão de pesquisa: quais os estabelecimentos de saúde credenciados no SUS que utilizam plantas medicinais e fitoterápicos como PICS estão cadastrados no CNES? Dessa maneira, objetiva-se verificar os estabelecimentos de saúde credenciados no Sistema Único de Saúde e no Cadastro Nacional de Estabelecimentos de Saúde, que utilizam plantas medicinais e fitoterápicos como Práticas Integrativas e Complementares.

\section{Método}

Trata-se de uma pesquisa que utiliza procedimentos da análise documental, o que possibilita explanar e esclarecer a questão/problema em consonância com o objetivo do 
pesquisador. Um documento é uma importante ferramenta de acesso para a compreensão do contexto social, dos conceitos e para o favorecimento de uma observação do passado, além de propiciar a visualização do processo de maturação ou de evolução dos indivíduos, grupos, conceitos, conhecimentos, comportamentos, mentalidades e práticas. ${ }^{14}$

$\mathrm{Na}$ primeira etapa da análise documental foi realizada a localização dos documentos pertinentes e avaliada sua credibilidade; assim, foram localizados os estabelecimentos que ofertam as PICS, sejam eles SUS ou não SUS, ambulatorial ou hospitalar recorrendo ao site http://cnes.datasus.gov.br/, leis, portarias e resoluções brasileiras referentes às PICS, ${ }^{9,11-12}$ realizada no mês de maio de 2018. Os documentos foram selecionados seguindo os critérios: pertinência do conteúdo ao objetivo do estudo e confiabilidade por se tratarem de documentos oficiais do MS. Os documentos referentes aos estabelecimentos que ofertam as PICS, resoluções, leis e portarias, constituem-se documentos públicos passíveis de serem pesquisados mediante análise documental. ${ }^{14}$

Assim, de posse desses documentos, foi realizada a análise documental preliminar, de acordo com as cinco orientações: análise do contexto, autores, autenticidade e confiabilidade do texto, natureza do texto, conceitos-chave e lógica interna do texto. ${ }^{12} \mathrm{Com}$ relação ao contexto das PICS, ressalta-se que a legitimação e a institucionalização destas práticas de atenção à saúde tiveram início na década de 1980, após a criação do SUS, ${ }^{4}$ ratificada posteriormente em 2006 e somada em 2017 e 2018 a outras PICS sendo, atualmente, são descritas e reconhecidas 39 PICS no Brasil..$^{9,11-12}$ No que se refere à autenticidade e à confiabilidade do material analisado, os documentos constituem-se em registros no CNES das PICS, leis, portarias e resoluções, ou seja, são documentos autênticos e confiáveis, analisados na íntegra. No cadastro constam informações de estabelecimentos de saúde cadastrados na esfera nacional pelo campo de consulta de serviços de práticas integrativas e complementares, código 134, no qual pode ser escolhido o estado, o município, o tipo de serviço, o serviço especializado, a classificação do 
serviço e o tipo de atendimento (ambulatorial ou hospitalar e SUS ou não SUS) as resoluções, leis e portarias que conceituam as 39 PICS, a sua aplicabilidade, políticas de inserção nos sistemas de saúde público e/ou privado.

$\mathrm{Na}$ última etapa, procedeu-se à análise dos documentos quanto à sua lógica interna, recorrendo-se à de conteúdo coerente a documental. ${ }^{14} \mathrm{~A}$ análise de conteúdo é um conjunto de técnicas de análise das comunicações verbal e não verbal que visa por meio de procedimentos sistemáticos e objetivos de descrição do conteúdo das mensagens para obter indicadores, qualitativos ou não, que permitam a inferência de conhecimentos relativos às condições de produção/recepção dessas mensagens.É considerada uma das técnicas de análise de conteúdo, cuja operacionalidade se distingue em três etapas: a pré-análise, a exploração do material, o tratamento dos resultados, a inferência e as interpretações. ${ }^{15}$

Há que se destacar que esta pesquisa se isenta do Termo de Consentimento Livre e Esclarecido (TCLE) por seu caráter documental. Assim, como os documentos analisados nesta pesquisa foram extraídos de sites oficiais, as questões que tangenciam os aspectos éticos foram respeitadas, considerando que as informações expostas traduzem o que tratam os dados encontrados.

\section{Resultados}

Os dados obtidos no presente estudo demonstram que dos 5.570 municípios brasileiros, ${ }^{16}$ 126 apresentam algum serviço que utilize a fitoterapia como prática integrativa e complementar. $\mathrm{Na}$ busca não foi possível encontrar a opção de pesquisa em separado das plantas medicinais e dos fitoterápicos. Cabe destacar que há distorções vocabulares em relação ao termo, pois em 2006 a PNPIC 9 trouxe a fitoterapia não englobando as plantas medicinas, o que vai de encontro ao cadastro nacional que tem somente o registro da fitoterapia, a qual corretamente contém as plantas medicinais e os fitoterápicos. 
Tabela 1 - Número de Estados por regiões brasileiras que utilizam a fitoterapia como prática integrativa e complementar credenciada no Sistema Único de Saúde. Brasil, 2018

\begin{tabular}{lcc}
\hline Região & Fitoterapia & Total \\
PICs & 8 & 8 \\
\hline Nordeste & 4 & 4 \\
Sudeste & 3 & 3 \\
Sul & 3 & 3 \\
Centro-oeste & 3 & 3 \\
Norte & $\mathbf{2 1}$ & $\mathbf{2 1}$ \\
\hline Total &
\end{tabular}

Fonte: Cadastro Nacional de Estabelecimentos de Saúde. Brasil, 2018

Cabe mencionar que as plantas medicinais, embora estejam citadas na política como um cuidado, isoladamentenão aparecem como prática terapêutica de serviço no cadastro do CNES. Outro aspecto relevante a ser destacado, é que em todas as regiões do Brasil há municípios que cadastraram fitoterapia no CNES, sendo que o Nordeste é a região de maior implementação (38,11\%), seguido pelo Sudeste (19,05\%), Sul (14,28\%), Centro-Oeste (14,28\%) e Norte $(14,28 \%)$.

Apesar de a política ter sido aprovada em 2006, foi possível observar que apenas 2,26\% dos municípios brasileiros oficializaram o serviço de fitoterapia no SUS. Assim, diferentes questionamentos são possíveis em relação à oficialização do serviço de fitoterapia dentro das PICS: os gestores implementaram a ação na rede de cuidado, mas não conseguem cadastrar o serviço?; Os gestores não aderiram ao serviço de fitoterapia?; Ou ainda, nos registros do CNES há incompletudes que não reconhecem as informações dos municípios que oferecem o serviço fitoterápico? Estes questionamentos tornam-se ainda mais pertinentes quando existe somente a opção de fitoterapia para cadastro dentre as PICS, não desfragmentando plantas medicinais de fitoterápicos. 
Contatou-se que não há um esclarecimento entre os serviços de saúde cadastrados que ofertam somente as plantas medicinais ou os fitoterápicos, bem como não se distingue no cadastro os serviços de saúde que ofertam as duas práticas juntas (plantas medicinais e os fitoterápicos). Este registro inadequado pode gerar confusão acerca do termo correto a ser utilizado e o que realmente o serviço de saúde está ofertando a população. No momento, observam-se contradições nos números e carências de dados para emitir um ponto de vista substanciado que traga argumentos para que apresente a realidade da implantação da fitoterapia nacional no SUS.

Nas informações do CNES em maio de 2018, estavam cadastrados 589 serviços de fitoterapia dentro das PICS (Tabela 2).

Tabela 2- Número total de serviços de saúde que ofereciam a fitoterapia como prática integrativa complementa. Brasil, 2018

\begin{tabular}{lcc}
\hline Região & Fitoterapia & Total \\
\hline Sudeste & 324 & 324 \\
Sul & 151 & 151 \\
Nordeste & 97 & 97 \\
Centro-oeste & 10 & 10 \\
Norte & 7 & 7 \\
\hline Total & $\mathbf{5 8 9}$ & $\mathbf{5 8 9}$ \\
\hline
\end{tabular}

Fonte: Cadastro Nacional de Estabelecimentos de Saúde. Brasil, 2018

Nota-se que os serviços de fitoterapia estão mais concentrados na região Sudeste, seguida da Sul e Nordeste.

De acordo com os dados analisados, os 589 serviços de fitoterapia SUS e não SUS cadastrados estão distribuídos entre 126 municípios brasileiros. Destes, 110 serviços estão na rede de atenção do SUS (Quadro 1), distribuídos em 563 serviços que prestam assistência no SUS utilizando a fitoterapia (11 também são hospitalares). 
Quadro 1 - Municípios brasileiros que possuem o serviço de fitoterapia no SUS, cadastrados no CNES. Brasil, 2018

\begin{tabular}{|c|c|c|c|c|c|c|}
\hline Região & Norte & Nordeste & $\begin{array}{l}\text { Centro- } \\
\text { Oeste }\end{array}$ & Sudeste & Sul & Total \\
\hline \multirow{17}{*}{$\begin{array}{l}\text { Municípios } \\
\text { com } \\
\text { fitoterapia }\end{array}$} & $\begin{array}{c}\text { Manacapuru } \\
\text { (AM) }\end{array}$ & $\begin{array}{l}\text { Olivenca } \\
\text { (AL) }\end{array}$ & $\begin{array}{l}\text { Planaltina } \\
\text { (DF) }\end{array}$ & Mariana (MG) & Londrina (PR) & \multirow[t]{17}{*}{110} \\
\hline & Xinguara (PA) & $\begin{array}{l}\text { Riachão do } \\
\text { Jacuípe (BA) }\end{array}$ & Guará (DF) & $\begin{array}{c}\text { Contagem } \\
\text { (MG) }\end{array}$ & Missal (PR) & \\
\hline & $\begin{array}{l}\text { Bannach } \\
\text { (PA) }\end{array}$ & $\begin{array}{l}\text { Mulungu } \\
\text { (CE) }\end{array}$ & $\begin{array}{l}\text { Recanto das } \\
\text { Emas (DF) }\end{array}$ & $\begin{array}{c}\text { São João Del } \\
\text { Rei (MG) }\end{array}$ & $\begin{array}{l}\text { Pato Bragado } \\
\text { (PR) }\end{array}$ & \\
\hline & & $\begin{array}{c}\text { Barbalha } \\
\text { (CE) }\end{array}$ & $\begin{array}{l}\text { Samambaia } \\
\text { (DF) }\end{array}$ & $\begin{array}{l}\text { Uberlândia } \\
\text { (MG) }\end{array}$ & $\begin{array}{l}\text { Bom Jesus do } \\
\text { Sul (PR) }\end{array}$ & \\
\hline & & $\begin{array}{l}\text { Fortaleza } \\
\text { (CE) }\end{array}$ & Gama (DF) & $\begin{array}{l}\text { Capetinga } \\
\text { (MG) }\end{array}$ & Ubiratã (PR) & \\
\hline & & $\begin{array}{l}\text { Viçosa do } \\
\text { Ceará (CE) }\end{array}$ & Vitória (ES) & $\begin{array}{l}\text { Queluzito } \\
\text { (MG) }\end{array}$ & $\begin{array}{c}\text { Porto Barreiro } \\
\text { (PR) }\end{array}$ & \\
\hline & & $\begin{array}{l}\text { Carnaubal } \\
\text { (CE) }\end{array}$ & Colatina (ES) & $\begin{array}{l}\text { Betim } \\
(\mathrm{MG})\end{array}$ & $\begin{array}{c}\text { Porto Alegre } \\
\text { (RS) }\end{array}$ & \\
\hline & & $\begin{array}{c}\text { João Pessoa } \\
\text { (PB) }\end{array}$ & $\begin{array}{c}\text { Baixo } \\
\text { Guandu (ES) }\end{array}$ & $\begin{array}{l}\text { Uberaba } \\
\text { (MG) }\end{array}$ & Ivoti (RS) & \\
\hline & & Picui (PB) & Cuiabá (MT) & $\begin{array}{l}\text { Juiz de Fora } \\
(\mathrm{MG})\end{array}$ & $\begin{array}{c}\text { Antônio Prado } \\
\text { (RS) }\end{array}$ & \\
\hline & & $\begin{array}{c}\text { Casserengue } \\
(\mathrm{PB})\end{array}$ & & $\begin{array}{c}\text { Águas } \\
\text { Formosas } \\
\text { (MG) }\end{array}$ & Gravataí (RS) & \\
\hline & & $\begin{array}{c}\text { Princesa } \\
\text { Isabel (PB) }\end{array}$ & & $\begin{array}{l}\text { Matozinhos } \\
\text { (MG) }\end{array}$ & Vacaria (RS) & \\
\hline & & $\begin{array}{l}\text { Juripiranga } \\
\text { (PB) }\end{array}$ & & $\begin{array}{l}\text { Nova Lima } \\
\text { (MG) }\end{array}$ & $\begin{array}{c}\text { Nova } \\
\text { Petrópolis (RS) }\end{array}$ & \\
\hline & & $\begin{array}{c}\text { Água Branca } \\
(\mathrm{AL})\end{array}$ & & $\begin{array}{c}\text { Teófilo Otoni } \\
(\mathrm{MG})\end{array}$ & $\begin{array}{c}\text { São Vicente do } \\
\text { Sul (RS) }\end{array}$ & \\
\hline & & $\begin{array}{c}\text { São Sebastião } \\
\text { do Umbuzeiro } \\
\text { (PB) }\end{array}$ & & $\begin{array}{c}\text { Delfinópolis } \\
\text { (MG) }\end{array}$ & Ijuí (RS) & \\
\hline & & Goiana (GO) & & Claraval (MG) & $\begin{array}{c}\text { Rio Grande } \\
\text { (RS) }\end{array}$ & \\
\hline & & $\begin{array}{l}\text { Recife } \\
(\mathrm{PE})\end{array}$ & & $\begin{array}{l}\text { Varzelândia } \\
\text { (MG) }\end{array}$ & $\begin{array}{l}\text { Boa Vista do } \\
\text { Cadeado (RS) }\end{array}$ & \\
\hline & & Jurema (PE) & & $\begin{array}{c}\text { Araguari } \\
(\mathrm{MG})\end{array}$ & Blumenau (SC) & \\
\hline
\end{tabular}


11 | Badke MR, Cogo SB, Ilha AG, Heisler EV, Schimith MD, Sacramento HT

\begin{tabular}{|c|c|c|c|c|c|c|}
\hline & & Petrolina (PE) & & $\begin{array}{c}\text { Belo Oriente } \\
\text { (MG) }\end{array}$ & $\begin{array}{c}\text { Florianópolis } \\
\text { (SC) }\end{array}$ & \\
\hline & & $\begin{array}{c}\text { Campo Maior } \\
\text { (PI) }\end{array}$ & & Ipatinga $(\mathrm{MG})$ & $\begin{array}{c}\text { Balneário } \\
\text { Picarras (SC) }\end{array}$ & \\
\hline & & $\begin{array}{c}\text { Bom Jesus } \\
(\mathrm{PI})\end{array}$ & & $\begin{array}{c}\text { São Sebastião } \\
\text { do Paraíso } \\
(\mathrm{MG})\end{array}$ & $\begin{array}{c}\text { Rancho } \\
\text { Queimado (SC) }\end{array}$ & \\
\hline & & Natal (RN) & & Louveira (SP) & $\begin{array}{c}\text { Leoberto Leal } \\
\text { (SC) }\end{array}$ & \\
\hline & & $\begin{array}{c}\text { Tenente } \\
\text { Laurentino } \\
\text { Cruz (RN) }\end{array}$ & & $\begin{array}{c}\text { Boa } \\
\text { Esperança do } \\
\text { Sul (SP) }\end{array}$ & Maravilha (SC) & \\
\hline & & Baraúna $(\mathrm{RN})$ & & $\begin{array}{c}\text { Duque de } \\
\text { Caxias (RJ) }\end{array}$ & $\begin{array}{c}\text { Vidal Ramos } \\
\text { (SC) }\end{array}$ & \\
\hline & & $\begin{array}{c}\text { Olho-D’Agua } \\
\text { do Borges } \\
(\mathrm{RN})\end{array}$ & & $\begin{array}{c}\text { Cachoeiras de } \\
\text { Macacu (RJ) }\end{array}$ & Imbituba (SC) & \\
\hline & & $\begin{array}{l}\text { Simão Dias } \\
\text { (SE) }\end{array}$ & & $\begin{array}{c}\text { Rio de Janeiro } \\
(\mathrm{RJ})\end{array}$ & $\begin{array}{l}\text { Salto Veloso } \\
\text { (SC) }\end{array}$ & \\
\hline & & Salgado (SE) & & Niterói $(\mathrm{RJ})$ & $\begin{array}{l}\text { Mirim Doce } \\
\quad(\mathrm{SC})\end{array}$ & \\
\hline & & Aracaju (SE) & & $\begin{array}{c}\text { Volta } \\
\text { Redonda (RJ) }\end{array}$ & Tubarão (SC) & \\
\hline & & & & Ubirajara (SP) & Princesa (SC) & \\
\hline & & & & $\begin{array}{c}\text { Petrópolis } \\
\text { (RJ) }\end{array}$ & $\begin{array}{c}\text { Rio das Antas } \\
\text { (SC) }\end{array}$ & \\
\hline & & & & Itaboraí (RJ) & $\begin{array}{c}\text { Ponte Serrada } \\
\text { (SC) }\end{array}$ & \\
\hline & & & & $\begin{array}{c}\text { São Paulo } \\
\text { (SP) }\end{array}$ & $\begin{array}{c}\text { Alfredo } \\
\text { Wagner (SC) }\end{array}$ & \\
\hline & & & & Registro (SP) & Pomerode (SC) & \\
\hline & & & & $\begin{array}{l}\text { Campinas } \\
\text { (SP) }\end{array}$ & $\begin{array}{l}\text { Rio Negrinho } \\
\text { (SC) }\end{array}$ & \\
\hline & & & & $\begin{array}{c}\text { Santa Branca } \\
\text { (SP) }\end{array}$ & $\begin{array}{c}\text { Piratuba } \\
\text { (SC) }\end{array}$ & \\
\hline & & & & $\begin{array}{l}\text { Ribeirão } \\
\text { Preto (SP) }\end{array}$ & $\begin{array}{c}\text { Presidente } \\
\text { Castelo Branco } \\
\text { (PR) }\end{array}$ & \\
\hline & & & & Cajamar (SP) & & \\
\hline Total & 3 & 27 & 9 & 36 & 35 & 110 \\
\hline
\end{tabular}


Com relação a distribuição dos serviços de fitoterapia cadastrados por região brasileira, percebe-se que a região Sudeste ocupa o primeiro lugar, com 36 municípios oferecendo ao menos um serviço de fitoterapia, seguido pelo Sul (35) e a região Nordeste (27). Juntas, as regiões Centro-oeste e Norte somam 12 municípios.

\section{Discussão}

A fitoterapia valoriza a prevenção da doença e a recuperação da saúde, pensando o ser humano em sua integralidade. Embora seja uma prática milenar, ainda possui um tímido crescimento no SUS. Para obter um panorama deste serviço, foram selecionados alguns estudos que trazem informações a respeito dos profissionais da saúde, gestores e usuários.

Em uma pesquisa realizada com diretores de unidades de saúde da família de diferentes profissões (enfermeiros, gestores hospitalares, gestores de saúde, cirurgiões-dentista, farmacêutico, assistente social e administrador) do município de São Luís (MA), observou-se que 81\% desconheciam as políticas públicas para a implantação da fitoterapia e 56\% não conheciam o PNPMF. ${ }^{17}$ A maioria (94\%), acreditava que a inserção da fitoterapia como estratégia na atenção primária traria benefícios à comunidade por ser mais uma opção na busca de promoção da saúde, podendo reduzir custos com medicamentos. ${ }^{17}$

Um estudo realizado com profissionais de saúde da atenção básica com o Programa de Saúde da Família (PSF) do município de Juiz de Fora (MG), constatou que a equipe apresenta interesse na utilização da fitoterapia como PICS, porém justifica a falta de incentivo dos gestores, a descontinuidade de projetos em trocas de gestão, a necessidade de capacitação e o desconhecimento dos profissionais sobre a utilização das terapias alternativas, como principais dificuldades para a efetiva implementação da fitoterapia nos contextos do serviço. ${ }^{18}$

Em estudo realizado na cidade de Porto Alegre (RS), identificou-se que a maioria dos profissionais de saúde desconhece qualquer uma das políticas sobre PICS e os que 
13 | Badke MR, Cogo SB, Ilha AG, Heisler EV, Schimith MD, Sacramento HT

demonstraram possuir conhecimento foi devido a busca pessoal de informações e participação em eventos. Ainda nesse estudo, os profissionais relatam que há incentivo, mas não há uma estratégia organizada para a oferta de PICS. ${ }^{19}$ Pesquisa realizada no município de Crato (CE), constatou que os enfermeiros foram unânimes a favor do uso de fitoterapia e de plantas medicinais, pois acreditam que é uma alternativa viável e benéfica que auxiliaria no cotidiano assistencial, favorecendo o paciente e o sistema de saúde e a redução de custos na atenção básica. $^{20}$

Em Caico (RN), um estudo realizado com os profissionais de saúde, aponta que o baixo nível de conhecimento é um fator que gera dificuldade para o uso de PICS. Os profissionais atribuem esse fato, principalmente, a ausência desse conteúdo durante a graduação nos cursos da área da saúde. ${ }^{21}$ Uma investigação menciona que a inclusão de tal prática é possível por meio da participação popular, que ocorre quando há oferecimento de palestras e oficinas de manipulação dos fitoterápicos. No mesmo, estudo citado a facilidade de acesso como aspecto importante para a sua inclusão no SUS. Ela traz como dificuldades a pequena quantidade de ações de incentivo ao plantio de espécies vegetais no entorno domiciliar e o pequeno número de profissionais de saúde com conhecimento suficiente para prescrever plantas medicinais e medicamentos fitoterápicos. ${ }^{7}$

Uma pesquisa sobre a percepção dos médicos e gestores sobre a implementação das PICS no SUS no município de Vitória (ES), verificou que estes valorizam e buscam conhecer e estudar a Fitoterapia, e desejam participar dos cursos referentes à prescrição de medicamentos e tratamentos fitoterápicos. Além disso, os médicos da APS percebem melhoras significativas nos pacientes tratados com fitoterápicos e defendem a integração da Fitoterapia nas escolas e comunidades para a melhoria da qualidade de vida dos usuários, visando à autonomia, ao autocuidado, o empoderamento e o protagonismo. ${ }^{10}$ 
Diante deste panorama, verifica-se a necessidade de motivar os gestores e profissionais da saúde em relação à qualificação, oferta e complementação de cuidados integrativos e fitoterápicos nos serviços municipais. A literatura supracitada evidencia que o serviço de fitoterapia contribui para a consolidação dos princípios e diretrizes do SUS, sendo uma terapia de interesse dos profissionais, gestores e usuários do sistema. Os estudos pesquisados apontam dificuldades que precisam ser superadas para a implementação deste serviço, como problemas de acesso, desconhecimento sobre a PNPIC e de como utilizá-la, e falta de incentivo governamental.

Destacam-se ainda como necessidade para a implementação desta terapia, a ampliação do conceito de saúde dos gestores e profissionais, a inclusão dos conteúdos das PICS na graduação, a qualificação na área, a avaliação de satisfação da população com os serviços, o apoio institucional, político e de infraestrutura e maior comprometimento dos gestores. Além do exposto, inclui-se nestas demandas a necessidade de ampliar pesquisas com enfoque macro e micro deste serviço.

No que diz respeito aos profissionais da saúde legalizados para prescrever fitoterápicos é importante ressaltar o envolvimento de aspectos legais como a autorização pelo conselho profissional que exige capacitação específica de seus profissionais para este fim e aspectos éticos, como a existência de embasamento da prescrição, demonstrando conhecimento adequado. $^{22}$

Através da Resolução do Conselho de Enfermagem (COFEN) n 197/1997, que resolve em seu Art. 1ํㅗㄹ Estabelecer e reconhecer as Terapias Alternativas como especialidade e/ou qualificação do profissional de Enfermagem e Art. $2^{2}$ para receber a titulação prevista no artigo anterior, o profissional de enfermagem deverá ter concluído e sido aprovado em curso reconhecido por instituição de ensino ou entidade congênere, com uma carga horária mínima de 360 horas. Na Resolução no 577/2018, ${ }^{23}$ é permitido ao enfermeiro se especializar em diversas 
15 | Badke MR, Cogo SB, Ilha AG, Heisler EV, Schimith MD, Sacramento HT

áreas, e entre elas destacam-se as PICS. Elas estão descritas, hierarquicamente, no item 30 da área 1 da resolução, sendo: acupuntura, fitoterapia, homeopatia, ortomolecular, terapia floral, reflexologia podal, reiki, yoga, toque terapêutico, musicoterapia, cromoterapia e hipnose. Dessa maneira, destaca-se a atuação de enfermagem na fitoterapia e nas demais PICS.

\section{Conclusão}

Ao verificar o panorama dos estabelecimentos de saúde credenciados para o uso de plantas medicinais e fitoterápicos como prática integrativa e complementar no SUS, primeiramente foi constatado uma não adequação do uso correto do termo fitoterapia sendo algumas vezes, sinônimo de plantas medicinais e, em outros momentos, representando significados distintos, o que pode dificultar as pesquisas sobre a temática e cadastros dos referidos estabelecimentos de saúde. Evidenciou-se também uma disparidade na distribuição destes serviços em municípios e regiões do país.

Mesmo com a implantação da Política Nacional de Práticas Integrativas e Complementares no SUS em 2006, são poucos os estabelecimentos de saúde cadastrados, não oportunizando o usuário ao acesso as terapias complementares e restringindo tratamentos à medicamentos sintéticos.

A impossibilidade de pesquisar no CNES separadamente das plantas medicinais de fitoterápicos e vice-versa, pode ser considerada uma limitação deste estudo. Os resultados deste estudo podem subsidiar e encorajar os gestores dos Estados, municípios e do Ministério da Saúde no planejamento, monitoramento e avaliação dos cadastros e do funcionamento destes serviços, com vistas ao fortalecimento das práticas integrativas e complementares no SUS.Cabe também aos profissionais de saúde, procurar conhecer as PICS e integrá-las no cotidiano dos serviços, no intuito de facilitar o acesso da população a outras formas terapêuticas, que não só a biomédica, gerando a oportunidade de escolha de serviço. 
Referente aos profissionais da saúde, enquanto enfermeiros, acredita-se no potencial de atuação de Conselho para que amplie o reconhecimento de novas terapias integrativas e complementares como especialidade e/ou qualificação do profissional de Enfermagem.

\section{Referências}

1. Badke MR. Significado do uso de plantas em práticas de autoatenção em situações de padecimento [tese]. Pelotas: Universidade Federal de Pelotas; 2017 [acesso em 2018 maio 20]. Disponível em: https://wp.ufpel.edu.br/pgenfermagem/files/2017/04/TESE-Marcio-Rossato-Badke.pdf

2. Mascarenhas MA, Jacobsen MS, organizadores. Práticas integrativas e complementares em saúde: fundamentos e aplicabilidades. Porto Alegre: Ed. Universitária Metodista IPA; 2017.

3. Monteiro SC. Farmacobotânica: aspectos teóricos e aplicação. Porto Alegre: Artmed; 2017.

4. Ministério da Saúde (BR), Secretaria de Atenção à Saúde, Departamento de Atenção Básica. Política nacional de práticas integrativas e complementares no SUS: atitude de ampliação de acesso [Internet]. $2^{\underline{a}}$ ed. Brasília (DF): Ministério da Saúde; 2013 [acesso em 2017 out 10]. Disponível em: http://bvsms.saude.gov.br/bvs/publicacoes/politica_nacional_praticas_integrativas_complementares.pdf

5. World Health Organization (WHO). Traditional Medicine Strategy 2002-2005 [Internet]. Geneva: World Health Organization; 2002 [acesso em 2017 out 09]. Disponível em: http://www.wpro.who.int/health_technology/book_who_traditional_medicine_strategy_2002_2005.pdf

6. Ministério da Saúde (BR). VIII Conferência Nacional de Saúde: relatório final [Internet]. Brasília (DF): Ministério da Saúde; 1986 [acesso em 2017 out 09]. Disponível em: http://bvsms.saude.gov.br/bvs/publicacoes/8_conferencia_nacional_saude_relatorio_final.pdf

7. Figueiredo CA, Gurgel IGD, Gurgel Júnior GD. A política nacional de plantas medicinais e fitoterápicos: construção, perspectivas e desafios. Physis (Rio J) [Internet]. 2014 [acesso em 2017 nov 15];24(2):381-400. Disponível em: http://www.scielo.br/pdf/physis/v24n2/0103-7331-physis-24-02-00381.pdf

8. Fernandes TM. Pesquisa em plantas medicinais: 30 anos de busca de credibilidade. In: Plantas medicinais: memória da ciência no Brasil [Internet]. Rio de Janeiro: Fiocruz; 2004 [acesso em 2018 jun 30]. p. 77-160. Disponível em: http://books.scielo.org/id/bg6yw/pdf/fernandes-9788575413487-04.pdf.

9. Brasil. Ministério da Saúde. Portaria n. 971, de 3 de maio de 2006. Aprova a Política Nacional de Práticas Integrativas e Complementares (PNPIC) no Sistema Único de Saúde [Internet]. 2006 [acesso em 2017 nov 15]. Diário Oficial da União, Brasília (DF); 2006 maio 04. Seção 1, p. 20-4. Disponível em: http://bvsms.saude.gov.br/bvs/saudelegis/gm/2006/prt0971_03_05_2006.html 
10. Sacramento HT. A implementação da Política Nacional De Práticas Integrativas e Complementares no município de Vitória-ES: percepção de médicos e gestores [dissertação]. Vitória (ES): Escola Superior de Ciências da Santa Casa de Misericórdia de Vitória; 2015 [acesso em 2018 jun 17]. Disponível em: http://www.emescam.br/arquivos/pos/stricto/dissertacoes/85_Henriqueta_Tereza_Sacramento.pdf

11. Brasil. Ministério da Saúde. Portaria n. 849, de 27 de março de 2017. Inclui a arteterapia, ayurveda, biodança, dança circular, meditação, musicoterapia, naturopatia, osteopatia, quiropraxia, reflexoterapia, reiki, shantala, terapia comunitária integrativa e Yoga à Política Nacional de Práticas Integrativas e Complementares [Internet]. 2017 [acesso em 2017 nov 17]. Diário Oficial da União, Brasília (DF); 2017 mar 28. Seção $1, \quad$ p. 68-9. Disponível em: http://189.28.128.100/dab/docs/portaldab/documentos/prt_849_27_3_2017.pdf

12. Brasil. Ministério da Saúde. Portaria n. 702, de 21 de março de 2018. Altera a Portaria de Consolidação n. 2/GM/MS, de 28 de setembro de 2017, para incluir novas práticas na Política Nacional de Práticas Integrativas e Complementares - PNPIC [Internet]. 2018 [acesso em 2018 maio 10]. Diário Oficial da União, Brasília (DF): 2018 mar 21. Seção 1, p. 74. Disponível em: http://bvsms.saude.gov.br/bvs/saudelegis/gm/2018/prt0702_22_03_2018.html

13. Ministério da Saúde (BR). Cadastro Nacional de Estabelecimentos de Saúde - CNES [Internet]. Brasília (DF); 2018 [acesso em 2018 maio 09]. Disponível em: http://datasus.saude.gov.br/sistemas-eaplicativos/cadastros-nacionais/cnes

14. Cellard A. A análise documental. In: Poupart J, Deslauries J-P, Groulx 1-H, Laperrière A, Mayer R, Pires A, organizadores. A pesquisa qualitativa: enfoques epistemológicos e metodológicos. $3^{\mathbf{a}}$ ed. Petrópolis: Vozes; 2012. p. 295-316.

15. Bardin L. Análise de conteúdo. Lisboa: Edições 70; 2016.

16. Instituto Brasileiro de Geografia e Estatística (IBGE). Novos mapas municipais do IBGE mostram que Brasil tem agora 5.570 municípios [Internet]. Rio de Janeiro; 2013 [acesso em 2017 nov 16]. Disponível em: https://agenciadenoticias.ibge.gov.br/2013-agencia-de-noticias/releases/14431-asi-novos-mapasmunicipais-do-ibge-mostram-que-brasil-tem-agora-5570-municipios.html

17. Araujo WRM, Silva RV, Barros CS, Amaral FMM. Inserção da fitoterapia em unidades de saúde da família de São Luís, Maranhão: realidade, desafios e estratégias. Rev Bras Med Fam Comunidade [Internet]. 2014 [acesso em 2017 nov 16];9(32):258-63. Disponível em: https://www.rbmfc.org.br/rbmfc/article/view/789

18. Barreto BB, Vieira RCPA. Percepção dos profissionais de saúde sobre a inserção da fitoterapia na atenção primária à saúde. Rev APS [Internet]. 2015 [acesso em 2017 nov 16]; 18(2):191-8. Disponível em: https://aps.ufj.emnuvens.com.br/aps/article/view/2226 
19. Müller TL. Práticas integrativas e complementares na atenção básica do Sistema Único de Saúde do município de Porto Alegre, RS: desafios atuais [dissertação ]. Porto Alegre: Universidade Federal do Rio Grande do Sul; 2016 [acesso em 2018 mar 20]. Disponível em: http://www.lume.ufrgs.br/handle/10183/157530

20. Sampaio LA, Oliveira DR, Kerntopf MR, Brito Júnior FE, Menezes IRA. Percepção dos enfermeiros da estratégia saúde da família sobre o uso da fitoterapia. REME Rev Min Enferm [Internet]. 2013 [acesso em 2017 dez 02];17(1):77-84. Disponível em: http://www.reme.org.br/artigo/detalhes/580

21. Varela DSS, Azevedo DM. Difficulties of health prefessionals facing the use of medicinal plants and fitotherapy. Rev Pesqui Cuid Fundam [Internet]. 2013 [acesso em 2017 dez 02];5(2):3588-600. Disponível em: http://www.seer.unirio.br/index.php/cuidadofundamental/article/view/2033/pdf_726

22. Oliveira AFP, Costa ICP, Andrade CG, Santos KFO, Anízio BKF, Brito FM. Fitoterapia na atenção básica: estudo com profissionais enfermeiros. Rev Pesqui Cuid Fundam [Internet]. 2017 [acesso em 2018 maio 15];9(2):480-7. Disponível em: http://www.seer.unirio.br/index.php/cuidadofundamental/article/viewFile/5449/pdf_1

23. Brasil. Ministério da Saúde. Resolução COFEN n. 577, de 5 junho de 2018. Atualiza, no âmbito do Sistema Cofen/Conselhos Regionais de Enfermagem, os procedimentos para Registro de Títulos de PósGraduação Lato e Stricto Sensu concedido a Enfermeiros e lista as especialidades [Internet]. 2018 [acesso em 2018 jul 02]. Disponível em: http://www.cofen.gov.br/resolucao-cofen-no-577-2018_63569.html

\section{Autor correspondente}

Marcio Rossato Badke

E-mail:marciobadke@gmail.com ou lapics@ufsm.br

Endereço Institucional: Universidade Federal de Santa Maria, Centro de Ciências da Saúde - Prédio 26, Sala 1305;

Faixa de Camobi, Km 09 - Campus Universitário Santa Maria/RS

CEP: $97105-900$

\section{Contribuições de Autoria}

\section{Marcio Rossato Badke}

- Concepção e planejamento do projeto de pesquisa; obtenção ou análise e interpretação dos dados; redação e revisão crítica.

\section{Silvana Bastos Cogo}

- Obtenção ou análise e interpretação dos dados; redação e revisão crítica. 
19 | Badke MR, Cogo SB, Ilha AG, Heisler EV, Schimith MD, Sacramento HT

\section{Aline Gomes Ilha}

- Obtenção ou análise e interpretação dos dados; redação e revisão crítica.

\section{Elisa Vanessa Heisler}

- Obtenção ou análise e interpretação dos dados; redação e revisão crítica.

\section{Maria Denise Schimith}

- Obtenção ou análise e interpretação dos dados; redação e revisão crítica.

\section{Henriqueta Tereza do Sacramento}

- Obtenção ou análise e interpretação dos dados; redação e revisão crítica.

\section{Como citar este artigo}

Badke MR, Cogo SB, Ilha AG, Heisler EV, Schimith MD, Sacramento HT. Panorama brasileiro dos serviços de plantas medicinais e fitoterápicos. Rev. Enferm. UFSM. 2019 [Acesso em: Anos Mês Dia];vol9 e64:1-19. DOI:https://doi.org/10.5902/2179769233655 